\title{
Qualität soll sich auszahlen
}

\section{Petra Seeburger}

Kommunikationsbeauftragte "Hausärzte Schweiz»

\author{
Der Schweizer Hausärzteverband wünscht sich eine Hausarztmedizin durch Fach- \\ ärzte für Allgemeine Innere Medizin. Die rund 800 in der Schweizer Grundver- \\ sorgung arbeitenden "Praktischen Ärzte» würde er daher gerne motivieren, einen \\ solchen Facharzttitel zu erlangen. Vorstandsmitglied Eva Kaiser und SIWF-Juristin \\ Barbara Linder erklären die Zusammenhänge im folgenden Beitrag, der in Frage- \\ und-Antwort-Form gestaltet ist.
}

Ein Schwerpunkt des Haus- und Kinderärzteverbands ist die Stärkung der Weiter- und Fortbildung. Die Forderung der Hausarztinitiative wurde im Masterplan aufgenommen und inzwischen im MedBG verankert. Wieso ist dies ein Anliegen der Standespolitik?

Eva Kaiser: 70\% aller medizinischen Behandlungen in der Schweiz erfolgen ohne Überweisung oder weiterführende Untersuchung durch Haus- und Kinderärzte. Je besser diese ausgebildet sind, um so besser ist die Qualität der ärztlichen Grundversorgung. Gerade bei den Hausärzten spielt darum die Weiterbildung zum Facharzt eine wichtige Rolle. Diese führt heutzutage über ein fünfjähriges Curriculum zum Titel «Allgemeine Innere Medizin (AIM)». Dem Verband ist wichtig, dass Hausärzte nicht nur in Spitä-

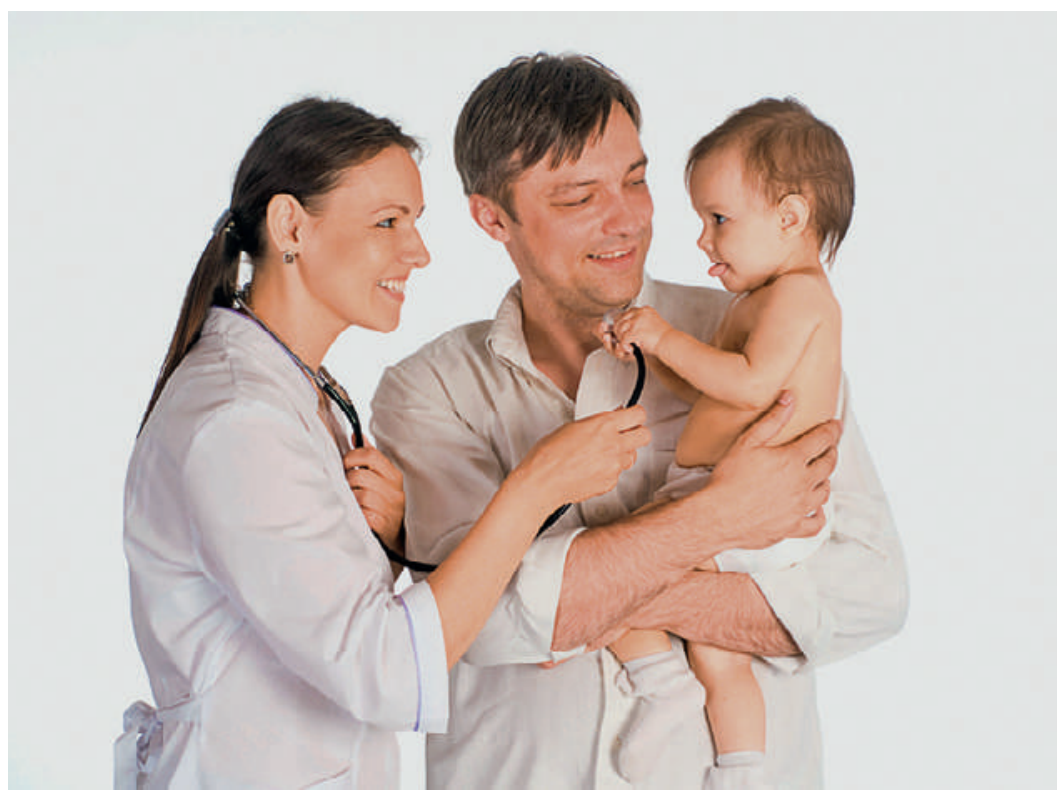

«Hausärzte Schweiz» wünscht sich ein eigenes Grundversorgerkapitel im TARMED, das nur für Haus- und Kinderärzte mit einem anerkannten Facharzttitel abrechenbar ist. lern, sondern auch in Praxen weitergebildet werden, wo sie später auch arbeiten. Deshalb wurden Praxisassistenzen als Elemente der Facharztweiterbildung etabliert und im MedBG integriert.

\section{Neben der Facharztausbildung AIM gibt es noch} eine Weiterbildung zum «Praktischen Arzt». Warum? Barbara Linder: Die dreijährige Weiterbildung zum Weiterbildungstitel «Praktischer Arzt» ist die Minimalanforderung für die selbständige Tätigkeit und den Erhalt einer Praxisbewilligung. In der Regel ist sie eine Art Zwischenschritt für den späteren Erwerb des Facharzttitels «Allgemeine Innere Medizin». Der «Praktische Arzt» wurde aber meist nur im Hinblick auf einen drohenden Zulassungstopp beantragt, um rechtzeitig eine Praxisbewilligung $\mathrm{zu}$ bekommen. Bedeutung hat der Titel «Praktischer Arzt» bei der Anerkennung von Allgemeinmedizinern aus dem EU-Raum.

\section{Wie ist das zu verstehen?}

Barbara Linder: In der EU-Richtlinie über die Anerkennung von Berufsqualifikationen haben alle Mitgliedstaaten der EU ihre Weiterbildungstitel notifiziert, damit diese gegenseitig anerkannt werden können, so auch in der Allgemeinmedizin. Wenige Länder wie beispielsweise Deutschland haben dafür eine fünfjährige Weiterbildung. Die Allgemeinmediziner der meisten europäischen Länder absolvieren hingegen nur eine dreijährige Weiterbildung. Im Rahmen der bilateralen Verträge hat die Schweiz für den äquivalenten europäischen Titel in Allgemeinmedizin nur den "Praktischen Arzt» notifiziert, der eine dreijährige Weiterbildung umfasst. Der frühere Facharzttitel Allgemeinmedizin war nie Gegenstand des bilateralen Abkommens. 


\begin{tabular}{llll}
\hline FMH-Erhebung & $\mathbf{2 0 1 3}$ & $\mathbf{2 0 1 2}$ & $\mathbf{2 0 1 1}$ \\
\hline Ärztinnen und Ärzte mit WB-Titel «Praktischer Arzt» im ambulanten Sektor & 835 & 743 & 648 \\
\hline Ärztinnen und Ärzte mit Facharzttitel «Allgemeine Innere Medizin» im ambulanten Sektor & 5929 & 5869 & 5800 \\
\hline
\end{tabular}

Quelle: www.fmh.ch/aerztestatistik (Berufstätige Ärzte nach Sektor/Hauptfachgebiet)

Offenbar macht diese Praxis Betroffenen Probleme?

Barbara Linder: EU-Fachärzte für Allgemeinmedizin werden in der Schweiz als "Praktischer Arzt» anerkannt. Für Fachärzte, die in ihrem Land bereits eine fünfjährige strukturierte Weiterbildung inklusive Facharztprüfung absolviert haben, ist dies unbefriedigend. Wer zusätzlich in der Schweiz den eidgenössischen Facharzttitel «Allgemeine Innere Medizin» erwerben will, muss belegen, dass seine Weiterbildung äquivalent ist oder diese ansonsten vervollständigen. Dies kann beim SIWF abgeklärt werden.

\section{Ausländer sind also gezwungen, einen Teil ihrer Weiterbildung zu wiederholen?}

Barbara Linder: Bei ausländischen Allgemeinmedizinern, die den Facharzttitel AIM erlangen wollen, aber nur eine dreijährige Weiterbildung nachweisen können, ist das nötig. Wer hingegen über einen fünfjährigen Titel verfügt, kann sich die im Ausland absolvierte Weiterbildung vollumfänglich anrechnen lassen, sofern es sich um gleichwertige und dem Weiterbildungsprogramm entsprechende Weiterbildung gehandelt hat. Dies ist aber nur möglich, wenn nach altem Programm "Allgemeinmedizin» beurteilt werden kann. Diese Möglichkeit steht nur denjenigen offen, die bis Ende 2015 sämtliche Be- dingungen des Programms erfüllt haben. Für alle anderen Antragssteller ist ab 2016 ausschliesslich das Weiterbildungsprogramm "Allgemeine Innere Medizin» anwendbar. Das bedeutet konkret, dass für die Erlangung eines Facharzttitels AIM in jedem Fall eine mindestens 18-monatige Weiterbildung an einer anerkannten Schweizer Weiterbildungsstätte absolviert werden muss. Ebenfalls muss die Facharztprüfung in der Schweiz abgelegt werden. Die deutschen Fachärzte für Allgemeinmedizin haben aber die Möglichkeit, anstelle des Titels «Praktischer Arzt» ihren ausländischen und formell durch die Medizinalberufekommission anerkannten Titel im Wortlaut und in der Landessprache des Ausstellungslandes mit Verweis auf das Herkunftsland zu tragen also «Facharzt für Allgemeinmedizin (D)».

\section{Der Berufsverband der Schweizer Haus- und Kinderärzte unterstützt eine fünfjährige Weiterbil- dung zum Facharzt. Wieso?}

Eva Kaiser: Das Bewusstsein, dass wir für die Schweiz eine gute medizinische Grundversorgung mit dem Hausarzt im Zentrum brauchen, ist dank unseren gemeinsamen Anstrengungen im ganzen Land gestiegen. Die Hausärzte sind «Spezialisten fürs Komplexe». Wenn wir weiterhin wollen, dass der allergrösste Teil

\section{Informationen zum Erwerb des eidgenössischen Facharzttitels «Allgemeine Innere Medizin» für Ärztinnen und Ärzte mit einem im Ausland erworbenen Weiterbildungstitel "Allgemeinmedizin»}

Ärztinnen und Ärzte mit einem im EU-Raum erworbenen Titel in Allgemeinmedizin können in der Schweiz gestützt auf das bilaterale Abkommen Schweiz-EU als "Praktischer Arzt» / «Praktische Ärztin» anerkannt werden. Dies ist insbesondere für diejenigen Titelinhaber unbefriedigend, die im Herkunftsland dafür eine fünfjährige strukturierte Weiterbildung absolviert haben (z.B. Deutschland). Verbunden mit der Anerkennung ist auch die Ausschreibung des Titels. Wer über einen anerkannten Allgemeinmediziner-Titel verfügt, kann sich entweder als "Praktischer Arzt» / "Praktische Ärztin» auskünden oder aber die Bezeichnung des erworbenen Titels im Wortlaut und der Landessprache des Ausstellungsstaates unter Angabe des Herkunftslandes verwenden, z.B. Facharzt für Allgemeinmedizin (D).

Wer zusätzlich in der Schweiz den eidgenössischen Facharzttitel Allgemeine Innere Medizin erwerben möchte, kann über die Website des SIWF eine Standortbestimmung bei der Geschäftsstelle des SIWF beantragen. Folgende Punkte sind zu beachten:
- Den Titel kann noch nach altem Weiterbildungsprogramm "Allgemeinmedizin» erwerben, wer dessen Bedingungen bis Ende 2015 erfüllt hat. Diesfalls kann die gesamte im Ausland absolvierte Weiterbildung berücksichtigt werden, soweit sie dem Weiterbildungsprogramm entspricht und gleichwertig ist.

- Für die Überprüfung durch die Titelkommission des SIWF müssen die Belege gemäss Merkblatt auf der Website des SIWF eingereicht werden.

- Wer nicht sämtliche Bedingungen des alten Weiterbildungsprogramms "Allgemeinmedizin» bis zum 31.12.2015 erfüllt, wird nach neuem Weiterbildungsprogramm «Allgemeine Innere Medizin" beurteilt und muss in jedem Fall mindestens 18 Monate Weiterbildung an einer für Allgemeine Innere Medizin anerkannten Weiterbildungsstätte in der Schweiz absolvieren. Ebenfalls ausgewiesen werden muss die bestandene Facharztprüfung in der Schweiz.

Weiterführende Informationen: www.siwf.ch

Barbara Linder, SIWF 


\section{med. pract. $\neq$ Praktischer Arzt}

Kennen Sie den Unterschied? Wahrscheinlich nicht, und dann sind Sie nicht die/der Einzige. Der Titel "med. pract.» wird oft mit dem Weiterbildungstitel "Praktischer Arzt» verwechselt. "med. pract.» ist eine Bezeichnung, die von Medizinern verwendet wurde, die zwar ein Staatsexamen gemacht haben, aber keine Doktor-Promotion haben. Bis zum Inkrafttreten der bilateralen Verträge mit der EU war der Doktor-Titel Voraussetzung für den Erwerb von damals noch privatrechtlichen Facharzttiteln. Die Bezeichnung «Praktischer Arzt» darf nur verwenden, wer diesen dreijährigen Weiterbildungstitel erworben hat oder über eine formelle Anerkennung eines Allgemeinmediziner-Titels aus einem der Mitgliedstaaten der EU verfügt. Wer weder über einen Weiterbildungstitel noch über eine Dissertation verfügt, sollte sich - wegen der oben beschriebenen Verwechslungsgefahr nach den heutigen Empfehlungen nicht mehr als «med. pract.» auskünden (alternativ denkbar z.B. «dipl. Arzt» / «dipl. Ärztin» oder «dipl. med.»).

Korrespondenz: Petra Seeburger Kommunikationsbeauftragte "Hausärzte Schweiz" Effingerstrasse 2

CH-3011 Bern petra.seeburger[at] hausaerzteschweiz.ch der Gesundheitsprobleme abschliessend vom Hausarzt behandelt werden kann, ohne dass eine Weiterweisung an eine Spezialdisziplin erfolgt, reicht eine dreijährige Weiterbildung einfach nicht aus. Schweizer Fachärzte für "Allgemeine Innere Medizin» haben eine sehr gut fundierte und breite Weiterbildung. Nach ihrem Facharztexamen sind sie zu einer kontinuierlichen Fortbildung verpflichtet, für die sie von ihrer Fachgesellschaft regelmässig rezertifiziert werden. Diese hohe Qualität zu erhalten, ist dem Verband sehr wichtig.

\section{Der Hausärzteverband fordert, dass Hausärzte mit} Facharzttitel mehr verdienen sollen, als ihre Kollegen, die «Praktische Ärzte» sind?

Eva Kaiser: Es soll für unseren Nachwuchs in den Hausarztpraxen interessanter sein, einen Facharzttitel zu tragen, als möglichst rasch mit der Weiterbildung fertig zu sein. Darum wünscht sich «Hausärzte Schweiz» ein eigenes Grundversorgerkapitel im TARMED, das nur für Haus- und Kinderärzte mit einem anerkannten Facharzttitel (AIM oder KJM) abrechenbar ist und durch das die fachärztliche Tätigkeit der Haus- und Kinderärzte dauerhaft korrekt abgebildet ist. Motiv dafür ist nicht, einzelne Kolleginnen und Kollegen zu übervorteilen, sondern auch in der Hausarztmedizin einen qualitativen Kompetenzstandard umzusetzen. Die Weiterbildungsqualität soll sich auf diese Weise auch finanziell auszahlen.

\section{Dass sich Ihre Kollegen "Praktische Ärzte» daran stören, verstehen Sie?}

Eva Kaiser: Dass sich vor allem die ausländischen Kollegen mit einer fünfjährigen Weiterbildung inklusive absolvierter Facharztprüfung daran stören, dass sie durch ein solches Kapitel ausgegrenzt würden, ist begreiflich. Wir bedauern auch, dass ihr Titel bei den Abkommen mit der EU unter die Räder gekommen ist. Unser Ziel ist jedoch die ausgewiesene Qualität in der ärztlichen Grundversorgung. Wir sind überzeugt, dass der finanzielle Anreiz dazu ein guter Weg ist.

\section{Was heisst das für gut qualifizierte Hausärzte mit dem Titel «Praktischer Arzt»?}

Eva Kaiser: Wir empfehlen allen Hausärztinnen und Hausärzten, einen Facharzttitel AIM zu erlangen. Bis Ende 2015 gelten Übergangsregeln, nach denen das alte Curriculum der SGAM noch gilt und die äquivalente Weiterbildung im Ausland vollständig anerkannt werden kann. Ich rechne damit, dass deutsche Fachärzte für Allgemeinmedizin die Voraussetzungen für den Schweizer Titel AIM oftmals schon erfüllen oder dass die Hürde zur Erlangung nur gering ist. Sie sollten es versuchen bzw. in Erfahrung bringen, was ihnen für den Schweizer Titel noch genau fehlt. Auf der Homepage des SIWF stehen die wichtigsten Informationen, bei Bedarf steht das SWIF auch beratend zur Seite. Wir sind stolz auf die Qualität unseres Gesundheitssystems, das davon lebt, dass beim Hausarzt zentral die Fäden zusammenlaufen. Diese Qualität gilt es zu erhalten, darum brauchen wir «Fachärzte» als Hausärzte. Und «Facharzt» sein soll sich auszahlen. 